\title{
Die Schweiz und der Weltgipfel über die Informationsgesellschaft: Herausforderungen und Defizite
}

Fabrice Boulé

\section{CpenEdition}

\section{Journals}

Electronic version

URL: http://journals.openedition.org/sjep/559

DOI: $10.4000 /$ sjep.559

ISSN: 1663-9677

Publisher

Institut de hautes études internationales et du développement

Printed version

Date of publication: 1 novembre 2003

Number of pages: 157-162

ISSN: 1660-5926

\section{Electronic reference}

Fabrice Boulé, «Die Schweiz und der Weltgipfel über die Informationsgesellschaft: Herausforderungen und Defizite », Schweizerisches Jahrbuch für Entwicklungspolitik [Online], 22-2 | 2003, Online erschienen am: 10 Juni 2010, abgerufen am 07 September 2020. URL : http://journals.openedition.org/sjep/559 ; DOI : https://doi.org/10.4000/sjep.559 


\title{
Die Schweiz und der Weltgipfel über die Informationsgesellschaft: Herausforderungen und Defizite
}

\author{
Fabrice Boulé*
}

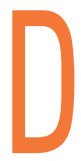

er Weltgipfel über die Informationsgesellschaft (WSIS) treibt die Schweiz in die Enge. Die Verfechter der Genfer Kandidatur sahen eine politische Gelegenheit für die UNO-Stadt und eine verstärkte internationale Rolle der Schweiz bei ausschlaggebenden Themen in den nächsten zehn Jahren voraus. Es ist das erste Mal, dass die internationale Gemeinschaft sich auf solch hoher Ebene mit Kommunikation und Information befasst. Um die Unterschiede zu anderen Gipfeln zu betonen, wurden neue Regeln angekündigt: Die Gesellschaft und der Privatsektor müssen sich den politischen Entscheidungsträgern spürbar annähern können. Die jetzt offizielle Tendenz wird von der UNO seit mehreren Jahren gefördert. Aber Genf 2003 ist ein entscheidender Test für diese Zusammenarbeit, denn der Gipfel soll eine gemeinsame Vision der Informationsgesellschaft von morgen ausdrücken, die von den Staaten, der Zivilgesellschaft und den wirtschaftlichen Akteuren geteilt wird.

Im langen diplomatischen Tauziehen um die Vorbereitungen eines solchen Gipfels ist es sowohl eine Chance als auch ein Risiko, Gastland zu sein: Man hat einen gewissen Manövrierraum, um den Weg aufzuzeigen und Vorschläge zu machen, aber man wird auch weitgehend für einen möglichen Misserfolg verantwortlich gemacht. Nach ihrem UNO-Beitritt im September 2002 muss die Schweiz nun den Vereinten Nationen beweisen, dass sie fähig ist, einen verlockenden Gipfel vorzubereiten. Wird er zum Erfolg, erhält die Schweiz als neuer Akteur mehr Gehör auch bei anderen Themen, die ihr wichtig sind: Förderung der Menschenrechte, der menschlichen Sicherheit (Kampf gegen Personenminen, den Handel mit leichten Waffen, die Rekrutierung von Kindersoldaten), Einstehen für die Menschenrechte (die Schweiz will 2007 für die Ad-hoc-Kommission kandidieren), Umweltschutz (Einstehen für das Vorsichts- und das Verursacherprinzip).

Woran misst man den Erfolg? An einer unfehlbaren Logistik? Das ist denjenigen, die den Gipfel vorbereiten, sehr wichtig, denn es geht ums Image. An Zahl und Format der anreisenden Staats- und Regierungschefs? Zweifellos, denn das Echo in den Medien hängt stark von deren Anwesenheit ab. Aber die VIPs kommen nur, wenn das Programm attraktiv, politisch gewichtig und gut für das Image ist. Gleichzeitig darf aber niemand in Bedrängnis gebracht werden. Die Kommunikationsinfrastrukturen und die Art der Informationen müssen theoretisch ein Beitrag zur Erreichung der von der UNO in ihrer Millenniumserklärung festgehaltenen Ziele sein: Abbau der Armut, Gesundheitsdienste und Bildung für

* Journalist bei InfoSud. 
alle, gute Regierungsführung, Aufwertung der Rolle der Frau usw. Es geht also darum, den grössten gemeinsamen Nenner zwischen Regierungen, Privatsektor und Zivilgesellschaft zu finden, indem mit sehr heiklen Themen wie Recht auf freie Meinungsäusserung, Zugang zur Information als öffentliches Gut oder auch Regeln des geistigen Eigentums usw. jongliert wird. Seit Anfang Jahr macht sich Marc Furrer, Direktor des Bundesamtes für Kommunikation (BAKOM) und Leiter des Gipfels von Seiten der Schweiz für eine offene und innovative Zusammenkunft stark: Zivilgesellschaft und Privatsektor müssen aktiv teilnehmen und mitentscheiden, damit der Gipfel zu einem Erfolg wird.

1992 hatte die UNO in Rio lange zum Voraus einen "Mister Erdgipfel“ bestimmt. Maurice Strong umgab sich mit Dutzenden von Mitarbeitenden. Diese verhalfen dem Konzept der „nachhaltigen Entwicklung“ zum Durchbruch, von dem 1987 im Brundtland-Bericht erstmals die Rede war. Rio war ein Erfolg, weil vor dem Gipfel ein „Geist von Rio“ entstand. Für Genf fehlt so etwas. Es wurde keine Führungspersönlichkeit gesucht, um einen Geist von Genf aufzubauen und den WSIS zu einem „Rio der Information“ zu machen. Die Schweiz hat keine eigene Vision der Informationsgesellschaft von morgen und konnte deshalb keine eigenen Leute und auch keine möglichen Verbündeten von Bedeutung mobilisieren, die sich hinter eine solche Flagge hätten stellen könnten. Es ging nicht darum, dass der kaum in die UNO aufgenommene Frosch einem Ochsen gleichen sollte, es ging vielmehr darum, Visionäre zu versammeln, Ideen auszubrüten und den Eingeladenen ein stimulierendes Menü vorzusetzen.

Schauen wir zurück auf den Mai 2002 und die Konferenz zur Vorbereitung des Gipfels, die in Bamako, der Hauptstadt Malis, stattfand. Die Schweiz finanzierte dieses erste Treffen zusammen mit der Europäischen Union und den frankophonen Ländern. Dabei stellte Walter Fust, Chef der Direktion für Entwicklung und Zusammenarbeit (DEZA) der Schweiz, seine Vision vor: Nicht eine technische Konferenz, sondern ein grosser Gipfel der sozialen Kommunikation. Und vor allem nicht einfach ein Schönheitswettbewerb unter den Staaten. Aber bereits damals wollte man sich nicht zu sehr in den Vordergrund stellen, zu sehr exponieren. „Achtung, der WSIS ist kein Schweizer, sondern ein UNO-Gipfel“, warnte Fust. „Viele afrikanische Länder verwechseln das. Die UNO muss bei den Staaten für ihn werben, und leider müssen wir feststellen, dass sie damit noch nicht begonnen hat." Anderthalb Jahre später hat die UNO diese Aufgabe noch immer nicht richtig angepackt. Und die Schweiz verpasst, aus Vorsicht oder Unentschlossenheit, eine Chance, die Dinge in die Hand zu nehmen.

Sicher gibt es verschiedene Gründe, warum man sich nicht stärker für den WSIS einsetzt. Sie hängen sowohl mit dem internationalen Kontext von 2003 wie mit den verschlungenen Wegen zwischen UNO, Bund und Kantonen zusammen, welche die Vorbereitungen komplizieren:

$\checkmark$ Das „Made in Switzerland“ mit der Aufgabenteilung zwischen den verschiedenen Bundesämtern, das Fehlen einer klaren politischen Linie, das andauernde Desinteresse und das fehlende Bewusstsein dessen, was auf dem Spiel steht, lasten schwer. Im Frühling 2003 versuchte man, diese Hindernisse abzubauen, doch da war schon viel Zeit verloren gegangen. 
... PENDANT LE SOMMET,

LA SUISSE SERA LE

NOMBRIL DE LA TOILE!

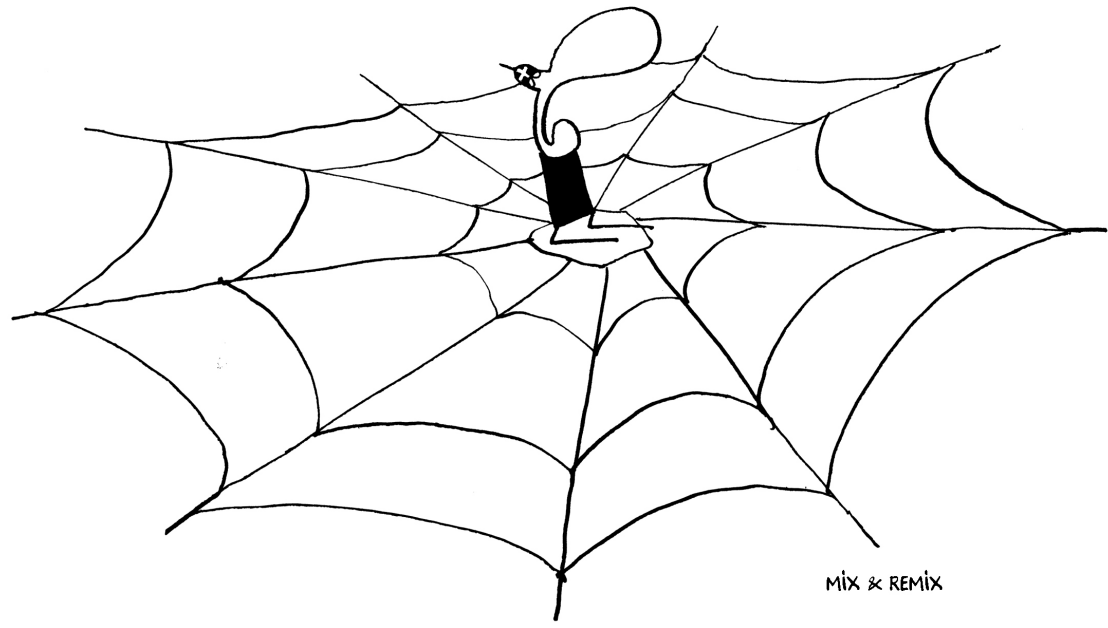

... Während des Gipfels ist die Schweiz der Nabel des Webs!

$\checkmark$ Der ganze „Kampf gegen den Terrorismus“ nach dem 11. September 2001, der Krieg in Afghanistan und der Krieg in Irak waren dem Nachdenken über die Verbreitung des Wissens im 21. Jahrhundert auch nicht förderlich.

- Die Unentschlossenheit Yoshio Utsumis, des Generalsekretärs der Internationalen Fernmeldeunion (UIT), in Bezug auf die Kandidaturen von Genf und Tunis führte dazu, dass alle über zehn Monate lang in den Startblöcken verharrten. Tunesien wollte seit mehreren Jahren einen Informationsgipfel durchführen. Dazu hatte das Land 1998 am UIT-Kongress in Minneapolis in einer Resolution eine Machbarkeitsstudie für einen solchen Gipfel verlangt. Die Verabschiedung dieser Resolution öffnete den Weg zum WSIS. Zu Beginn war allerdings nur ein UIT-Gipfel für 2002 vorgesehen: für die Regierungen und die Industrie, aber ohne Zivilgesellschaft. 2001 forderte dann eine Resolution der UNO-Generalversammlung die volle Teilnahme der Zivilgesellschaft.

$\square$ Die Vergabe der zweiten Phase an Tunis stellt uns natürlich vor ein Problem. Das Land glänzt durch die totale Unterdrückung der freien Meinungsäusserung. Gerade als die Prepcom (Vorbereitungskomitee) zum zweiten Mal tagte, wurden mehrere Internetsurfer von den tunesischen Behörden verhaftet, weil sie politisch inkorrekte Websites abgefragt hatten. Die Opposition verlangt von der Regierung Signale der Öffnung, um die Vergabe von 2005 
auch zu verdienen, glaubt aber selber nicht recht daran. Immerhin dürfte die Fortsetzung des WSIS in Tunis den tunesischen Verfechtern der Menschenrechte eine erstklassige Tribüne verleihen, um sich Gehör zu verschaffen.

$\square$ Diese erzwungene Partnerschaft zwischen der Schweiz und Tunesien führte manchmal zu abstrusen Vorkommnissen. Anfang 2003 hatte der frühere malische Minister Adama Samassekou, der die Vorbereitungsarbeiten leitet, die Idee, vor Dezember nochmals alle zusammenzurufen: Die Präsidenten der Gastländer des Gipfels und der Länder, welche die regionalen Vorbereitungskonferenzen durchführten, sollten sich in Genf treffen. Einige Schwarzseher sahen sofort ein Bild mit einem Händedruck zwischen Pascal Couchepin und dem tunesischen Präsidenten Ben Ali im Zentrum, um die Informationsgesellschaft von morgen zu verkaufen. Damit war das Projekt begraben. Offiziell sind sich Bern und Tunis natürlich in allem einig.

- Die UIT, die von der UNO den Auftrag hat, die Vorbereitung des WSIS zu koordinieren, ist eine technische Agentur. Sie hat keine Erfahrungen in multilateralen politischen Verhandlungen. Sie hat ihren Sitz in Genf und organisiert da jeweils die Telekomausstellung. Ausserdem arbeitet die UIT eng mit Privatfirmen zusammen, hatte aber bisher noch nie mit der Zivilgesellschaft zu tun. Viele Schweizer WSIS-Akteure klagen die UIT der Untätigkeit, wenn nicht gar der Verhinderungsmanöver an, um die Kontrolle des WSIS nicht abgeben zu müssen.

$\square$ Viele Schweizer Akteure beklagen sich auch über das tiefe finanzielle Engagement der UIT. Bern seinerseits hat rund 20 Millionen Franken freigegeben. Davon sind rund 15 Millionen für Sicherheit, Logistik und Organisation des Gipfels vorgesehen (die Stellen zweier Mitglieder im Exekutivsekretariat, jene des Delegierten des Bundesrates, die Kosten für die Organisation der Prepcoms). Zwischen 5 und 6 Millionen Franken sollten in Parallelanlässe fliessen, welche von der Direktion für Entwicklung und Zusammenarbeit (DEZA), dem Staatssekretariat für Wirtschaft (seco), den Bundesämtern für Kultur, Statistik usw. organisiert werden. Der Kanton Genf wiederum zahlt 2 bis 3 Millionen Franken: rund eine Million für Logistik, Sicherheit und Protokoll, und zwischen einer und 2 Millionen für den Teil der Zivilgesellschaft, das bedeutet zwei Stellen für die Zivilgesellschaft im Büro des Gipfels für rund zwei Jahre sowie Logistikkosten für die Präsenz und die Beteiligung von Nichtregierungsorganisationen.

- Die wirtschaftliche Krise im Telekom- und Informatikbereich erschwert die Mobilisierung der Unternehmen in diesem Sektor. Ihre abwartende Haltung schwächt den tripartiten Charakter des WSIS (Regierungen plus internationale Organisationen - Zivilgesellschaft - Privatsektor).

$\checkmark$ Warum wurde die Leitung dem Bundesamt für Kommunikation (BAKOM) und nicht dem Departement für auswärtige Angelegenheiten (EDA) übergeben? In Bern wurde das offenbar nicht wirklich diskutiert. Da das BAKOM Mitglied der UIT ist, ist es zwar einigermassen logisch, dass es die Leitung übernimmt. Es koordiniert auch den interdepartementalen Ausschuss Informationsgesellschaft (IDAIG), der mit der Leitung der Aktivitäten des Bundes im Bereich Informations- und Kommunikationstechnologien (IKT) betraut ist. Es führt jedes Jahr eine Bestandesaufnahme zuhanden des Bundesrates 
durch. Als die Rollen Anfang Sommer 2001 verteilt wurden, steckten das EDA und Joseph Deiss mitten in der Kampagne für den UNO-Beitritt. Die Energie und die nötigen Finanzen für die Vorbereitung des WSIS fehlten also damals. Auf jeden Fall riss sich das EDA nicht darum, wodurch die Möglichkeiten einer frühen weltweiten Mobilisierung durch die Schweizer Diplomatie im Hinblick auf den Gipfel verloren gingen. Die in der Förderung der IKT zugunsten der Entwicklung sehr aktive DEZA wäre sicher interessiert gewesen, aber sie liess nichts verlauten. Der WSIS ist nicht eigentlich ein Entwicklungsgipfel. Ausserdem hatte die DEZA keine Lust auf das Risiko, am Schluss die Rechnung für eine Versammlung von Telekomtechnikern bezahlen zu müssen. Und schliesslich ist für sie die Durchführung eines WSISParallelanlasses wichtiger, bei dem in Genf die besten Beispiele der Anwendung der IKT für die soziale und wirtschaftliche Entwicklung vorgestellt werden (ICT4D).

$\square$ Ein Star aus Afrika hat in letzter Minute abgesagt. Seit langem hatten zwei Genfer WSIS-Akteure - Alain Clerc, der die Abteilung Zivilgesellschaft im Exekutivsekretariat leitet, und der ehemalige Staatsrat Guy-Olivier Segond, UIT-Sonderbotschafter für den WSIS - eine Sonderrolle für ihren Freund Alpha Oumar Konaré vorgesehen, der bis 2002 Präsident Malis war. Dieser verzichtete aber auf den Vorsitz der Gipfelvorbereitungen, denn die Leitung der neuen Afrikanischen Union (vormals OAU) war ihm wichtiger. Schliesslich wurde der ehemalige Erziehungsminister Malis, Adama Samassekou, für den Posten ernannt. Das Fehlen von Präsident Konaré, dem Mann, der alle Gemeinden seines Landes per Internet verbinden wollte, führte natürlich dazu, dass das Ganze an Sichtbarkeit verlor. Der in letzter Minute ernannte Ersatzmann arbeitet sehr aktiv und effizient, ist aber weniger präsent in den Medien. In Mali präsidiert er die afrikanische Akademie der Sprachen, welche ein Inventar der vielen in Westafrika gesprochenen Sprachen erstellt. Dies ist sicher eine interessante Erfahrung für einen Gipfel, der sich ja auch mit der sprachlichen und kulturellen Vielfalt auf dem Web befassen will.

Die Achse Genf-Bamako oder Genf-Afrika spielte bei der Entstehung des WSIS eine grosse Rolle. Nelson Mandela brachte das Thema bei der Eröffnung der Telecom 1995 aufs Tapet. Er bemerkte, dass die neuen Technologien nicht nur den Nord-Süd-Graben nicht verkleinerten, sondern vielmehr die „Infoarmen“ noch stärker von den „Inforeichen“ trennten. Im Jahr darauf organisierte Genf eine Konferenz über Afrika und die neuen Technologien. Alpha Oumar Konaré überzeugte als Ehrengast die Vertreter der Zivilgesellschaft. Ein Traum war geboren: Könnten nicht die Regierungen mit den Gewerkschaften, den Frauen, den Jungen, der Forschung und den Unternehmensleitungen auf gleichberechtigter Ebene diskutieren? Die Fondation du devenir unter Leitung von Alain Clerc organisierte daraufhin mit finanzieller Unterstützung durch Genf und die DEZA die Konferenz „Bamako 2000: Internet, les passerelles du développement". Vertreterinnen und Vertreter aus rund 40 Staaten sowie Abgeordnete der Zivilgesellschaft und des Privatsektors gaben eine gute Mischung ab. Auch die UIT war dabei. Anschliessend verlangte sie von Genf, das 1998 beschlossene, aber seither vernachlässigte Projekt eines Gipfels an die Hand zu nehmen. Damit trat auch die Zivilgesellschaft auf den Plan. Die Abteilung Zivilgesellschaft wird vom Kanton Genf finanziert. 
Die Genfer wiederholen es immer wieder: Der WSIS ist der erste Gipfel einer neuen Generation, der auf der lebendigen Kraft der wirklichen Gesellschaft aufbaut - dem Engagement der Bürgerinnen und Bürger und der Dynamik des Privatsektors. Die Regierungen können nicht mehr alles allein entscheiden. Das gilt vor allem im Zusammenhang mit Information und Kommunikation. Aber im Juli 2002 gibt es eine kalte Dusche für die Zivilgesellschaft: An der Prepcom 1 wollten die Regierungen nichts von ihr wissen. Die Nichtregierungsorganisationen (NRO) im Vorzimmer wurden ungeduldig. Wurden sie betrogen? Die Versprechen waren sicher übertrieben und das Motto "Gipfel ohnegleichen“ vielleicht zu marktschreierisch. Es stimmt, die Zusammenarbeit der Staaten, der NRO und der Unternehmen an den UNO-Gipfeln wurde nicht in Genf erfunden. Sie ist seit Jahren im Gang. Die Entwicklung der staatlichen Mentalitäten verläuft zwar langsam, aber sicher. Von Gipfel zu Gipfel entwickeln sich die Verfahrensregeln weiter.

Danach ging es etwas besser. Am zweiten Vorbereitungstreffen im Februar 2003 konnten die Mitglieder der Zivilgesellschaft in den Arbeitsgruppen mitarbeiten. Sie konnten sogar Änderungen am Entwurf der Grundsatzerklärung und dem Aktionsplan vorschlagen. Den Regierungen steht es aber frei, diese Vorschläge, die in einem separaten, aber immerhin offiziellen Dokument festgehalten sind, anzunehmen oder abzulehnen. Gleichzeitig wurde ein Büro für die Zivilgesellschaft geschaffen, eine permanente Einrichtung im Hinblick auf den Gipfel, die danach sogar in Genf bleiben soll - wenn sie die nötigen Finanzen erhält. „Ein organisiertes und als legitimer Ansprechpartner anerkanntes Büro für die Zivilgesellschaft könnte dem binären und sterilen Gegensatz zwischen den Diplomatenanlässen und den ,Demos “ auf den Strassen ein Ende setzen“, bemerkt Alain Clerc. Gleichzeitig macht auch die Schweizer Zivilgesellschaft vorwärts. Im Februar forderte eine Plattform aus Nichtregierungsorganisationen, Berufsverbänden und Medien Bern auf, aktiv zu werden. Die Zivilgesellschaft ist frustriert und glaubt, dass die innovative Dynamik des Gipfels gelitten hat. Sie droht mit einem Gegengipfel und verlangt, dass eine Reflexionsgruppe auf hoher Ebene eingesetzt wird, in der Abgeordnete der Bundesverwaltung, des Parlaments, der Zivilgesellschaft, der Medien und des Privatsektors Einsitz haben. Das Prinzip einer verstärkten Zusammenarbeit wird von Bern akzeptiert, doch werden die Forderungen der NRO etwas zurückgestutzt: Man sagt zwar Ja zu einer intensiveren Beratung, aber Nein zur institutionellen paritätischen Anerkennung der Zivilgesellschaft, deren Mitglieder nicht demokratisch gewählt sind. 